International Journal of Pure and Applied Mathematics

Volume 111 No. 2 2016, 179-188

ISSN: 1311-8080 (printed version); ISSN: 1314-3395 (on-line version)

url: http://www.ijpam.eu

doi: 10.12732/ijpam.v111i2.4



\title{
VAGUE VECTOR SPACE AND VAGUE MODULES
}

\author{
K.V. Rama Rao ${ }^{1 \S}$, Amarendra Babu V. ${ }^{2}$ \\ ${ }^{1}$ Department of Mathematics \\ S.R.R. and C.V.R Degree and P.G College \\ Vijayawada, A.P, INDIA \\ ${ }^{2}$ Department of Mathematics \\ Achraya Nagarjuna University \\ Nagarjuna Nagar, 522510, INDIA
}

\begin{abstract}
We introduce the concept of vague vector spaces by linking the vague set and vector spaces. Also we define vague modules. We discuss some properties both vague vector space and vague module.
\end{abstract}

AMS Subject Classification: 20N25, 08A72, 03E72

Key Words: vague set, vector space, vague vector space and vague module

\section{Introduction}

The concept of fuzzy set was introduced by Zadesh since then this idea been applied to other algebraic structures such as groups, rings etc. Meanwhile the dificiency of fuzzy sets is also attract attention. Such as fuzzy set is single function, it does not express the evidence of supporting and opposing. Based on this reason, the concept of vague set introduced by Gau.W.L and Buehrer.D.J[5] in 1993. Vague sets as a extension of fuzzy sets, the idea of vague sets is that the membership of every element can be divided into two aspects including supporting and opposing. The notion of fuzzy groups defined by Rosen Field[7]is

\footnotetext{
Received: $\quad$ June 29, 2016

Revised: $\quad$ November 18, 2016

Published: December 11, 2016

$\S_{\text {Correspondence author }}$
}

(c) 2016 Academic Publications, Ltd. url: www.acadpubl.eu 
the first application of fuzzy set theory in algebra. Ranjit Biswas[2]initiated the study of vague algebra by studying vague groups. In $[3,4]$, we introduced the notions of vague additive groups, vague rings and vague fields.

In this paper, as an extension of our mentioned work, we introduce the notions of vague vector space and vague module. Also we discuss some of their properties.

\section{Preliminaries}

In this section we given some definitions and state some results for later use.

Definition 1. [5]A vague set $A$ in the universe of discourse $X$ is characterzied by two membership functions given by

1. A truth membership function $t_{A}: X \rightarrow[0,1]$ and

2. A false membership function $f_{A}: X \rightarrow[0,1]$,

Where $t_{A}(x)$ is a lower bound of the grade of membership of $x$ derieved from the "evidence for $x "$, and $f_{A}(x)$ is a lower bound on the negation of $x$ derived from the "evidence against $x$ " and $t_{A}(x)+f_{A}(x) \leq 1$. Thus the grade of membership of $x$ in the vague set $A$ is bounded by subinterval $\left[t_{A}(x), 1-f_{A}(x)\right]$ of $[0,1]$. The vague set $A$ is written as $A=\left\{\left\langle x,\left[t_{A}(x), f_{A}(x)\right]\right\rangle / x \in X\right\}$. Where the interval $\left[t_{A}(x), 1-f_{A}(x)\right]$ is called the vague value of $x$ in the vague set $A$ and denoted by $V_{A}(x)$.

Definition 2. [5] $A$ vague set $A$ of a universe $X$ with $t_{A}(x)=0$ and $f_{A}(x)=1$ for all $x \in X$, is called the zero vague set of $X$.

Definition 3. [5] $A$ vague set $A$ of a universe $X$ with $t_{A}(x)=1$ and $f_{A}(x)=0$ for all $x \in X$, is called the unit vague set of $X$.

Definition 4. [9] Let $A$ be a vague set $A$ of a universe $X$ with vague valueV $V_{A}$. Then the image of $A, f[A]$ is the vague set in $Y$ with vague value $V_{A}$ defined by

$$
V_{f[A]}(y)=\left\{\begin{array}{l}
\sup _{z \in f^{-1}(y)} \text { if } f^{-1}(y) \neq \phi, \\
0 \text { if otherwise, }
\end{array}\right.
$$

for all $y \in Y$, where $f^{-1}(y)=\{x / f(x)=y\}$.

Definition 5. [3]Let $(X,+)$ be a group. A vague set $A$ of $X$ is called a vague additive group (briefly VAG) of $X$ if the following condition is satisfies:

1. $V_{A}(x+y) \leq \max \left\{V_{A}(x), V_{A}(y)\right\}$, for all $x, y \in X$; 
2. $V_{A}(-x) \leq V_{A}(x)$, for all $x \in X$.

Definition 6. [4]Let $X$ be a ring and $R$ be a vague set of $X$. Then $R$ is called a vague ring of $X$ if the following conditions are satisfies:

1. $V_{R}(x+y) \leq \max \left\{V_{R}(x), V_{R}(y)\right\}$, for all $x, y \in X$;

2. $V_{R}(-x) \leq V_{R}(x)$, for all $x \in X$;

3. $V_{R}(x y) \geq \min \left\{V_{R}(x), V_{R}(y)\right\}$, for all $x, y \in L$.

Definition 7. [4]Let $X$ be a field and $F$ be a vague set of $X$. Then $F$ is called a vague feild of $X$ if the following conditions are satisfies:

1. $V_{F}(x+y) \leq \max \left\{V_{F}(x), V_{F}(y)\right\}$, for all $x, y \in X$ :

2. $V_{F}(-x) \leq V_{F}(x)$, for all $x \in X$;

3. $V_{F}(x y) \geq \min \left\{V_{F}(x), V_{F}(y)\right\}$, for all $x, y \in L$ :

4. $V_{F}\left(x^{-1}\right) \geq V_{F}(x)$, for all $x \in X$.

\section{Vague Vector Space}

In this section, we introduce the notion of Vague vector space and investigate some of their properties.

Definition 8. Let $V$ be a vector space over a feild $F$ and $A$ be a vague set of $V$. Then $A$ is a vague vector space of $V$ if the following properties are hold:

1. $V_{A}(x+y) \leq \max \left\{V_{A}(x), V_{A}(y)\right\}$, for all $x, y \in V$;

2. $V_{A}(a x) \leq V_{A}(x)$, for all $a \in F$ and $x \in V$;

3. $V_{A}(0)=0, x \in V$.

Example 9. Let $V=\{\alpha, \beta, \gamma, \delta\}$ be a set of vectors and $F$ be a Galio's field $G(F, 2)$, where $\alpha=(0,0), \beta=(1,0), \gamma=(0,1), \delta=(1,1)$ and $F=\{0,1\}$. Vector addition defined on $V$ as folows:

Clearly $\left(V,+_{2}\right)$ is an abelian group. Scalar multiplication defined as follows:

$$
c\left(a_{1}, a_{2}\right)=\left(c a_{1}, c a_{2}\right)
$$




\begin{tabular}{|c|c|c|c|c|}
\hline$+{ }_{2}$ & $\alpha$ & $\beta$ & $\gamma$ & $\delta$ \\
\hline$\alpha$ & $\alpha$ & $\beta$ & $\gamma$ & $\delta$ \\
\hline$\beta$ & $\beta$ & $\alpha$ & $\delta$ & $\gamma$ \\
\hline$\gamma$ & $\gamma$ & $\delta$ & $\alpha$ & $\beta$ \\
\hline$\delta$ & $\delta$ & $\gamma$ & $\beta$ & $\alpha$ \\
\hline
\end{tabular}

for all $c \in F$ and $a_{1 /}, a_{2} \in V$. Clearly scalar multiplication is well defined. Then $V$ is a vector space over $G(F, 2)$.

Let $A$ be a vague set of $V$ defined by

$$
t_{A}(x)=\left\{\begin{array}{l}
0 \quad \text { if } \quad x=\alpha \\
0.7 \quad \text { if } \quad x=\beta \\
0.8 \quad \text { if } \quad x=\gamma, \delta
\end{array}\right.
$$

and

$$
f_{A}(x)=\left\{\begin{array}{l}
1 \quad \text { if } \quad x=\alpha \\
0.2 \quad \text { if } \quad x=\beta \\
0.1 \quad \text { if } \quad x=\gamma, \delta
\end{array}\right.
$$

Then $A$ is a vague vector space.

Example 10. Let $V=\left\{e_{1}, e_{2}, e_{3}, e_{4}, e_{5}, e_{6}, e_{7}, e_{8}, e_{9}\right\}$ be a set of vectors and $F\left(I_{3},+_{3}, \times_{3}\right)$ be a field, where $e_{1}=0, e_{2}=1, e_{3}=2, e_{4}=x, e_{5}=2 x$, $e_{6}=x+1, e_{7}=x+2, e_{8}=2 x+1, e_{9}=2 x+2$ and $I_{3}=\{0,1,2\}$. Vector addition defined on $V$ as folows:

\begin{tabular}{|c|c|c|c|c|c|c|c|c|c|}
\hline$+_{3}$ & $e_{1}$ & $e_{2}$ & $e_{3}$ & $e_{4}$ & $e_{5}$ & $e_{6}$ & $e_{7}$ & $e_{8}$ & $e_{9}$ \\
\hline$e_{1}$ & $e_{1}$ & $e_{2}$ & $e_{3}$ & $e_{4}$ & $e_{5}$ & $e_{6}$ & $e_{7}$ & $e_{8}$ & $e_{9}$ \\
\hline$e_{2}$ & $e_{2}$ & $e_{3}$ & $e_{1}$ & $e_{6}$ & $e_{8}$ & $e_{7}$ & $e_{4}$ & $e_{9}$ & $e_{5}$ \\
\hline$e_{3}$ & $e_{3}$ & $e_{1}$ & $e_{2}$ & $e_{7}$ & $e_{9}$ & $e_{4}$ & $e_{6}$ & $e_{5}$ & $e_{8}$ \\
\hline$e_{4}$ & $e_{4}$ & $e_{6}$ & $e_{7}$ & $e_{5}$ & $e_{1}$ & $e_{8}$ & $e_{9}$ & $e_{2}$ & $e_{3}$ \\
\hline$e_{5}$ & $e_{5}$ & $e_{8}$ & $e_{9}$ & $e_{1}$ & $e_{4}$ & $e_{2}$ & $e_{3}$ & $e_{6}$ & $e_{7}$ \\
\hline$e_{6}$ & $e_{6}$ & $e_{7}$ & $e_{4}$ & $e_{8}$ & $e_{2}$ & $e_{9}$ & $e_{5}$ & $e_{3}$ & $e_{1}$ \\
\hline$e_{7}$ & $e_{7}$ & $e_{4}$ & $e_{6}$ & $e_{9}$ & $e_{3}$ & $e_{5}$ & $e_{8}$ & $e_{1}$ & $e_{2}$ \\
\hline$e_{8}$ & $e_{8}$ & $e_{9}$ & $e_{5}$ & $e_{2}$ & $e_{6}$ & $e_{3}$ & $e_{1}$ & $e_{7}$ & $e_{4}$ \\
\hline$e_{9}$ & $e_{9}$ & $e_{5}$ & $e_{8}$ & $e_{3}$ & $e_{7}$ & $e_{1}$ & $e_{2}$ & $e_{4}$ & $e_{6}$ \\
\hline
\end{tabular}

Clearly $\left(V,+_{3}\right)$ is an abelian group. Then $V$ is a vector space with respect to adition and multiplication of polynomials. Let $A$ be a vague set of $V$ defined 
by

$$
t_{A}(x)=\left\{\begin{array}{l}
0 \quad \text { if } \quad x=e_{1} \\
0.1 \quad \text { if } \quad x=e_{2}, e_{3} \\
0.2 \quad \text { if } \quad x=e_{4}, e_{5}, e_{6}, e_{7}, e_{8}, e_{9}
\end{array}\right.
$$

and

$$
f_{A}(x)=\left\{\begin{array}{l}
1 \quad \text { if } \quad x=e_{1} \\
0.8 \quad \text { if } \quad x=e_{2}, e_{3} \\
0.7 \quad \text { if } \quad x=e_{4}, e_{5}, e_{6}, e_{7}, e_{8}, e_{9}
\end{array}\right.
$$

Then $A$ is a vague vector space.

Theorem 11. If $A$ is a vague vector space of a vector space $V$ over a field $F$. Then $V_{A}(\lambda x)=V_{A}(x)$ for all $0 \neq \lambda \in F$.

Proof. For all $x \in V$, we have $V_{A}(\lambda x) \leq V_{A}(x)$, for some $0 \neq \lambda \in F$.

Also for all $x \in V$, we have $V_{A}(x)=V_{A}\left(\lambda^{-1} \lambda x\right) \leq V_{A}(\lambda x)$. That is $V_{A}(x) \leq$ $V_{A}(\lambda x)$.

Hence $V_{A}(\lambda x)=V_{A}(x)$.

Theorem 12. Let $V$ be a vector space over the field $F$ and $A$ is a vague subset of $V$. Then $A$ is a vague vector space of $V$ if and only if

$$
V_{A}(a x+b y) \leq \max \left\{V_{A}(x), V_{A}(y)\right\}
$$

for all $x, y \in V$ and $a, b \in F$.

Proof. Suppose that $A$ is a vague vector space of $V$, we have

$$
V_{A}(a x) \leq V_{A}(x)
$$

and

$$
V_{A}(b y) \leq V_{A}(y)
$$

for all $x, y \in V$ and $a, b \in F$.

Hence $V_{A}(a x+b y) \leq \max \left\{V_{A}(x), V_{A}(y)\right\}$.

Conversely suppose that if $a=b=1$. We have

$$
V_{A}(x+y) \leq \max \left\{V_{A}(x), V_{A}(y)\right\}
$$

Also if $b=0$, we have $V_{A}(a x)=V_{A}(a x+0 . x) \leq \max \left\{V_{A}(x), V_{A}(0)\right\}$.

Hence $V_{A}(a x) \leq V_{A}(x)$ for all $x \in V$ and $a \in F$. 
Theorem 13. The intersection of a family of vague vector spaces is a vague vector space.

Proof. Let $\left\{A_{i} / i \in I\right\}$ be a family of vague vector spaces of a vector space $V_{F}$.

If $x, y \in F$ and $\lambda \in F$, then

$$
\begin{aligned}
V_{A}(x+y) & =\operatorname{Sup}_{i \in I} V_{A}(x+y) \\
& \leq \operatorname{Sup}_{i \in I}\left\{\max V_{A}(x), V_{A}(y)\right\} \\
& =\max \left\{\sup _{i \in I} V_{A}(x), \sup _{i \in I} V_{A}(y)\right\}
\end{aligned}
$$

also

$$
\begin{aligned}
V_{A}(\lambda x) & =\operatorname{Sup}_{i \in I} V_{A}(\lambda x) \\
& \leq \operatorname{Sup}_{i \in I}\left\{\max V_{A}(x)\right\} \\
& \leq \max \left\{\operatorname{Sup}_{i \in I} V_{A}(x)\right\} \\
& =V_{A}(x) .
\end{aligned}
$$

Theorem 14. Let $V_{1}$ and $V_{2}$ be two vector spaces over a field $F$ and $f$ be a linear transformation of $V_{1}$ in to $V_{2}$. Let $A$ be a vague vector space of $V_{2}$. Then the inverse image $f^{-1}(A)$ of $A$ is a vague vector space of $V_{1}$.

Proof. Let $x, y \in V_{1}$ and $a, b \in F$, then

$$
\begin{aligned}
V_{f^{-1}(A)}(a x+b y) & =V_{A}(f(a x+b y)) \\
& =V_{A}[a f(x)+b f(y)] \\
& \leq \max \left\{V_{A} f(x), V_{A} f(x)\right\} \\
& =\max \left\{V_{f^{-1}(A)}(x), V_{f^{-1}(A)}(y)\right\}
\end{aligned}
$$

So $f^{-1}(A)$ is a vague vector space of $V_{1}$.

Theorem 15. Let $V_{1}$ and $V_{2}$ be two vector spaces over a field $F$ and $f$ be a linear transformation of $V_{1}$ in to $V_{2}$. Let $A$ be a vague vector space of $V_{1}$ that has the sup property then the image $f(A)$ of $A$ is a vague vector space of $V_{2}$.

Proof. Let $\alpha, \beta \in V_{2}$. If either $f^{-1}(\alpha)$ or $f^{-1}(\beta)$ is empty, then the inequality theorem

$$
V_{A}(a x+b y) \leq \max \left\{V_{A}(x), V_{A}(y)\right\}
$$


is satisfied, for all $x, y \in V$ and $a, b \in F$.

Suppose neither $f^{-1}(\alpha)$ nor $f^{-1}(\beta)$ is empty. Let $r_{0} \in f^{-1}(\alpha), s_{0} \in f^{-1}(\beta)$ and

$$
\begin{aligned}
& V_{A}\left(r_{0}\right)=\sup _{x \in f^{-1}(\alpha)} V_{A}(x), \\
& V_{A}\left(s_{0}\right)=\sup _{y \in f^{-1}(\beta)} V_{A}(y) .
\end{aligned}
$$

Then

$$
\begin{aligned}
V_{f(A)}(a \alpha+b \beta) & =\sup _{r \in f^{-1}(a \alpha+b \beta)} V_{A}(r) \\
& \leq \max \left\{V_{A}\left(r_{0}\right), V_{A}\left(s_{0}\right)\right\} \\
& =\max \left\{V_{f(A)}(\alpha), V_{f(A)}(\beta)\right\}
\end{aligned}
$$

So $f(A)$ is a vague vector space of $V_{2}$.

\section{Vague Module}

In this section, we introduce the notion of Vague module and investigate some of their properties.

Definition 16. Let $R$ be a ring and $M$ be a $R$ module. Then the vague set $A$ of $M$ is a vague module of $M$ if the following properties are hold:

1. $V_{A}(x+y) \leq \max \left\{V_{A}(x), V_{A}(y)\right\}$, for all $x, y \in M$;

2. $V_{A}(r x) \leq V_{A}(x)$, for all $r \in R$ and $x \in M$;

3. $V_{A}(0)=0,0 \in M$.

Example 17. Let $Z_{3}[i]=\{0,1,2, i, 1+i, 2+i, 2 i, 1+2 i, 2+2 i\}$ and $\left(Z_{3},+_{3}, \times_{3}\right)$ is a ring. Addition modulo3 table on $Z_{3}[i]$ as folows:

Clearly $\left(Z_{3},+_{3}\right)$ is an abelian group.

Multiplication modulo3 table on $Z_{3}[i]$ as folows:

Clearly $\left(Z_{3},+_{3}, \times_{3}\right)$ is a $R$ - module.

Let $A$ be a vague set of $Z_{3}$ defined by

$$
t_{A}(x)=\left\{\begin{array}{l}
0 \quad \text { if } \quad x=0 \\
0.1 \quad \text { if } \quad x=1,2 \\
0.2 \quad \text { if } \quad x=i, 1+i, 2+i, 2 i, 1+2 i, 2+2 i
\end{array}\right.
$$




\begin{tabular}{|c|c|c|c|c|c|c|c|c|c|}
\hline+3 & 0 & 1 & 2 & $i$ & $1+i$ & $2+i$ & $2 i$ & $1+2 i$ & $2+2 i$ \\
\hline 0 & 0 & 1 & 2 & $i$ & $1+i$ & $2+i$ & $2 i$ & $1+2 i$ & $2+2 i$ \\
\hline 1 & 1 & 2 & 0 & $1+i$ & $2+i$ & $i$ & $1+2 i$ & $2+2 i$ & $2 i$ \\
\hline 2 & 2 & 0 & 1 & $2+i$ & $i$ & $1+i$ & $2+2 i$ & $2 i$ & $1+2 i$ \\
\hline$i$ & $i$ & $1+i$ & $2+i$ & $2 i$ & $1+2 i$ & $2+2 i$ & 0 & 1 & 2 \\
\hline $1+i$ & $1+i$ & $2+i$ & $i$ & $1+2 i$ & $2+2 i$ & $2 i$ & 1 & 2 & 0 \\
\hline $2+i$ & $2+i$ & $i$ & $1+i$ & $2+2 i$ & $2 i$ & $1+2 i$ & 2 & 0 & 1 \\
\hline $2 i$ & $2 i$ & $1+2 i$ & $2+2 i$ & 0 & 1 & 2 & $i$ & $1+i$ & $2+i$ \\
\hline $1+2 i$ & $1+2 i$ & $2+2 i$ & $2 i$ & 1 & 2 & 0 & $1+i$ & $2+i$ & $i$ \\
\hline $2+2 i$ & $2+2 i$ & $2 i$ & $1+2 i$ & 2 & 0 & 1 & $2+i$ & $i$ & $1+i$ \\
\hline
\end{tabular}

\begin{tabular}{|c|c|c|c|c|c|c|c|c|c|}
\hline$\times_{3}$ & 0 & 1 & 2 & $i$ & $1+i$ & $2+i$ & $2 i$ & $1+2 i$ & $2+2 i$ \\
\hline 0 & 0 & 0 & 0 & 0 & 0 & 0 & 0 & 0 & 0 \\
\hline 1 & 0 & 1 & 2 & $i$ & $1+i$ & $2+i$ & $2 i$ & $1+2 i$ & $2+2 i$ \\
\hline 2 & 0 & 2 & 1 & $2 i$ & $2+2 i$ & $1+2 i$ & $i$ & $2+i$ & $1+i$ \\
\hline$i$ & 0 & $i$ & $2 i$ & 2 & $2+i$ & $2+2 i$ & 1 & $1+i$ & $1+2 i$ \\
\hline $1+i$ & 0 & $1+i$ & $2+2 i$ & $2+i$ & $2 i$ & 1 & $1+2 i$ & 2 & $i$ \\
\hline $2+i$ & 0 & $2+i$ & $1+2 i$ & $2+2 i$ & 1 & $i$ & $1+i$ & $2 i$ & 2 \\
\hline $2 i$ & 0 & $2 i$ & $i$ & 1 & $1+2 i$ & $1+i$ & 2 & $2+2 i$ & $2+i$ \\
\hline $1+2 i$ & 0 & $1+2 i$ & $2+i$ & $1+i$ & 2 & $2 i$ & $2+2 i$ & $i$ & 1 \\
\hline $2+2 i$ & 0 & $2+2 i$ & $1+i$ & $1+2 i$ & $i$ & 2 & $2+i$ & 1 & $2 i$ \\
\hline
\end{tabular}

and

$$
f_{A}(x)=\left\{\begin{array}{l}
1 \quad \text { if } \quad x=0 \\
0.8 \quad \text { if } \quad x=1,2 \\
0.7 \quad \text { if } \quad x=i, 1+i, 2+i, 2 i, 1+2 i, 2+2 i
\end{array}\right.
$$

Then $A$ is a vague $R$ - module.

Theorem 18. The intersection of a family of modules is a vague module.

Proof. Let $\left\{A_{i} / i \in I\right\}$ be a family of vague modules and $A=\bigcap_{i \in I} A_{i}$.

Then

$$
\begin{aligned}
V_{A}(x+y) & =\inf _{i \in I} V_{A}(a+b) \\
& \leq \inf _{i \in I}\left\{\max V_{A}(a), V_{A}(b)\right\} \\
& =\max \left\{V_{A}(x), V_{A}(y)\right\}
\end{aligned}
$$


also

$$
\begin{aligned}
V_{A}(r a) & =\operatorname{Sup}_{i \in I} V_{A}(r x) \\
& \leq \operatorname{Sup}_{i \in I}\left\{\max V_{A}(a)\right\} \\
& \leq \max \left\{\operatorname{Sup}_{i \in I} V_{A}(a)\right\} \\
& =V_{A}(a)
\end{aligned}
$$

Theorem 19. Let $M_{1}$ and $M_{2}$ be two $R$ - modules over the ring $R$ and $f$ be a linear transformation of $M_{1}$ in to $M_{2}$. Let $A$ be a vague module of $M_{2}$. Then the inverse image $f^{-1}(A)$ is a vague module of $M_{1}$.

Proof. Let $x, y \in M_{1}$ and $a, b \in R$, then

$$
\begin{aligned}
V_{f^{-1}(A)}(a x+b y) & =V_{A}(f(a x+b y)) \\
& =V_{A}[a f(x)+b f(y)] \\
& \leq \max \left\{V_{A} f(x), V_{A} f(x)\right\} \\
& =\max \left\{V_{f^{-1}(A)}(x), V_{f^{-1}(A)}(y)\right\} .
\end{aligned}
$$

So $f^{-1}(A)$ is a vague module of $M_{1}$.

Theorem 20. Let $M_{1}$ and $M_{2}$ be two $R$ - modules over a ring $R$ and $f$ be a linear transformation of $M_{1}$ in to $M_{2}$. Let $A$ be a vague module of $M_{1}$ then the image $f(A)$ of $A$ is a vague module of $M_{2}$.

Proof. Let $\alpha, \beta \in M_{2}$. If either $f^{-1}(\alpha)$ or $f^{-1}(\beta)$ is empty, then the inequality theorem

$$
V_{A}(a x+b y) \leq \max \left\{V_{A}(x), V_{A}(y)\right\}
$$

is satisfied, for all $x, y \in V$ and $a, b \in F$.

Suppose neither $f^{-1}(\alpha)$ or $f^{-1}(\beta)$ is empty. Let $r_{0} \in f^{-1}(\alpha), s_{0} \in f^{-1}(\beta)$ and

$$
\begin{aligned}
& V_{A}\left(r_{0}\right)=\sup _{x \in f^{-1}(\alpha)} V_{A}(x), \\
& V_{A}\left(s_{0}\right)=\sup _{y \in f^{-1}(\beta)} V_{A}(y) .
\end{aligned}
$$

Then

$$
\begin{aligned}
V_{f(A)}(a \alpha+b \beta) & =\sup _{r \in f^{-1}(a \alpha+b \beta)} V_{A}(r) \\
& \leq \max \left\{V_{A}\left(r_{0}\right), V_{A}\left(s_{0}\right)\right\} \\
& =\max \left\{V_{f(A)}(\alpha), V_{f(A)}(\beta)\right\} .
\end{aligned}
$$

So $f(A)$ is a vague module of $M_{2}$. 


\section{Conclusion}

In this paper, the concept of vague vector space and vague modules are introduced and some of properties are studied. It is hoped that these concepts will rise to the notations like vague normed linear spaces and vague inner product spaces, continuous mappings etc.

\section{References}

[1] L.A. Zadeh, Fuzzy sets, Information and Control, 8, (1965), 338-353.

[2] Rangit Biswas, Vague groups, Int. J. Comput. Congition, 4, No.2, (2006), 20 - 23.

[3] V. Amarendra Babu and K.V. Rama Rao, Vague additivr groups, Universal Journal of Applied Mathematics, 3, No. 3 (2015), 50 - 52.

[4] V. Amarendra Babu and K.V. Rama Rao, Vague rings and vague fields, Journal of Multidisciplinary Engineering Science and Technology, 2, No. 7, (2015), 1943 - 1945.

[5] W.L. Gau and D.J. Buehrer, Vague sets, IEEE Transactions on Systems, Man and Cybernetics, 23, No.20, (1993), 610-614.

[6] Hakimuddin Khan, Ahmad and Rangit Biswas, On Vague groups, International Journal of Computational Cognition, 5, No. 1,(2007), 27 - 30.

[7] Rosen Field, A fuzzy groups, Journal of Math. Anal and Appl., 35, (1971), 512 - 517.

[8] M. Derirci, Vague groups, Journal of Math. and Appl., 230, (1999), 142 - 156.

[9] T.Eswarlal and N. Rama Krishna, Vague fields and vague vector spaces, International Journal of Pure and Applied Mathematics, 94,No. 3, (2014), 295 - 305. 